\title{
Effective Antibiotic Stewardship Program: Controlling Antibiotic Resistance and Optimizing Drug Use in Ahmadi Hospital
}

\author{
Saud M. Alajmi ${ }^{*}$, Wadha N. Almarri ${ }^{2}$ \\ ${ }^{1}$ Ahmadi Hospital, Kuwait Oil Company, Ahmadi, Kuwait \\ ${ }^{2}$ Infection Prevention and Control Unit, Ahmadi Hospital, Kuwait Oil Company, Ahmadi, Kuwait \\ Email: ^alafjah@gmail.com
}

How to cite this paper: Alajmi, S.M. and Almarri, W.N. (2020) Effective Antibiotic Stewardship Program: Controlling Antibiotic Resistance and Optimizing Drug Use in Ahmadi Hospital. Journal of Analytical Sciences, Methods and Instrumentation, 10, $1-11$.

https://doi.org/10.4236/jasmi.2020.101001

Received: December 15, 2019

Accepted: January 19, 2020

Published: January 22, 2020

Copyright $\odot 2020$ by author(s) and Scientific Research Publishing Inc. This work is licensed under the Creative Commons Attribution International License (CC BY 4.0).

http://creativecommons.org/licenses/by/4.0/

\begin{abstract}
An Antimicrobial Stewardship Program (ASP) is established to promote cost effective and safe use of antimicrobial agents. Basically, this approach directs the management of antimicrobials in terms of selection of appropriate drugs, determination of administration routes and standardization of both medication dosage and treatment duration. The program involves, but is not limited to, Hospital Staff Education, Pharmacodynamics Dose Optimization, Computer-Assisted Decision Support Programs, Pharmacist-Driven Intravenous to Oral Switch Programs, Pharmacy Dosing Programs and Antibiotic Cycling. A study was conducted to determine effectiveness of the ASP in significantly improving patient outcomes (e.g. decrease morbidity and mortality from infection). This was undertaken by collecting restrospective data on patient length of hospital stay, bed turnover rate and antibiotic consumption over a period of 2 years since the ASP was initiated. Periodic data were then compared and interpreted based on its clinical, quality and financial implications in order to measure effectiveness of the program implementation.
\end{abstract}

\section{Keywords}

Antibiotics, Antibiotic Stewardship, Drug Resistance, Infectious Disease, Kuwait

\section{Introduction}

In addition to saving lives, antibiotics have played significant role in understanding major advances in medicine and surgery [1]. They have treated infections that require chemotherapy treatments, have chronic diseases including di- 
abetes, or rheumatoid arthritis, end-stage renal disease, or require complex surgeries such as organ transplants, joint replacements, or cardiac surgery [1]. Antibiotics have extended the expected life span by treating bacterial infections [2] [3]. The first antibiotic-penicillin-was very successful in treating the deathly infectious diseases. However, soon penicillin resistance emerged that threatened the advantages of penicillin [4]. To tackle the threatening diseases new beta-lactams were introduced that restored confidence of treating bacterial diseases [5]. This was followed by emergence of Methicillin resistance in Staphylococcus aureus (MRSA), development of Vancomycin for treating MRSA and then identification of Vancomycin-resistant cases [5].

Despite efforts to stem the upward trend of antibiotic resistance, the spread of multidrug-resistant organisms (MDROs) and MDRO-associated infections are continuously increasing in the hospital patients [6]. MDRO-associated infections has resulted in deterioration in clinical results, increase in health associated cost and risk of death. Cephalosporin-resistant Enterobacter infection has resulted in increase in hospital charges, length of stay and mortality [7].

Epidemiological studies have shown positive relationship among the antibiotic usage and evolution of antibiotic resistance bacterial strains [8]. Mutations, horizontal gene transfer and acquisition of plasmids provide multi-drug resistance to bacteria. The usage of antibiotics results in decimation of antibiotic susceptible bacteria, but antibiotic resistant bacteria grow and reproduce as a result of natural selection. The surviving bacteria share their genetic material to other strains making them resistant too. Diverse inheritance pattern and adaptive capabilities of the bacteria make them resistant whether antibiotics are used appropriately or inappropriately. This shed light on their careful and prudent use in order to slow down the process of antibiotic resistance development. It has been observed innumerable times that a bacteria that was resistant to an antibiotic acquires resistance [9]. Studies have suggested that more than $50 \%$ of antibiotic usage is inappropriate [10]. Even warning regarding development of resistant bacteria due to overuse or misuse of antibiotics has not resulted in decrease or appropriate use of antibiotic prescription [11]. In addition to overuse, inappropriate use of antibiotics has also resulted in speeding up the process of antibiotic resistance [12].

The terms which are used for antimicrobial stewardship programs may vary significantly such as antibiotic policies, antibiotic control program and antibiotic management programs. All these terms in general refer to a principal program with which emphasizes on change and controlled use of antimicrobial agents at any health care institution, it may use multiple strategies [13].

An ASP is established to control and regulate the cost effective and safe use of antimicrobial means and agents. Basically, this approach directs the appropriate management and selection of antimicrobials, administration routes, dosages of medicine and duration of treatment and therapy [14]. The ASP emphasizes on the use of right drug for appropriate time duration in right amount. In addition, above mentioned program controls the development of multidrug-resistant or- 
ganisms (MDROs), adversarial drug events, length of stay at hospital, collateral damages e.g. Clostridium difficile colitis development as well as reduces health care costs [15]. ASP plays a crucial role in controlling antimicrobial resistance because microbes perpetually become resistant due to the extensive exposure to antimicrobial agents. There are multiple strategies for improving the diagnosis of severe infections. Multidrug-resistant organisms are the main focus of this program which provides guidance about proper use of broad-spectrum therapies as well as avoiding unnecessary exposure to antibiotics. These diagnostic approaches result in rapid and immediate identification of causal organisms as well as improves the specificity of sepsis diagnosis. Antibiotic stewardship program has successfully been reported to reduce the incidence and colonization of $C$. difficile [16].

Optimum antimicrobial stewardship comprises choosing the most suitable drug at its best dosage and period of therapy to eliminate an infection while curtailing side effects and pressures of resistant strains adaptation [17]. The decision matrix utilized by physicians to advice antibiotics can guide as a conceptual framework. Making a suitable antibiotic option begins with awareness of infectious diseases and the organisms implicated in different infections, as well as identifying which species are resistant to a specific antibiotic and whether that antibiotic can enter the infection site. Therefore, in taking these decisions, both pharmacodynamic and pharmacokinetic awareness is vital [18]. Overall clinical experience, including renal function, drug reactions, and use of concurrent medications that can end in drug interactions are imperative concerns that must be taken into account in the decision to pick and use an antibiotic. Another important factor is the availability of a particular antibiotic in hospital setting. Microbiological surveillance or screening of susceptibility may play a key role in improving antibiotic selection. Empirical therapy usually involves the use of broadspectrum medications, but it is important to move to a narrower-activity medicine when the effects of available culture test indicate that such an approach is acceptable [19].

Antibacterial stewardship has 2 main methods, however, the best strategy is to apply both of them. The front-end or pre prescription approach that uses restrictive prescriptive authority. There are certain antimicrobials that are considered restricted and need earlier authorization for usage by all practitioners except a selected group of clinicians. Clinicians that are lacking the authority to prescribe a particular drug are required to contact the nominated antimicrobial steward and request for approval for that particular antimicrobial. The front-end approach provides advantage of targeting some antimicrobials depending on local resistance patterns and the hospital formulary [20] [21].

The back-end or postprescription approach to stewardship utilizes feedback and review. The antimicrobial steward reviews existing antibiotic orders and is responsible for recommending clinicians to continue, adjust, change, or discontinue the antibiotic usage depending upon the availability of microbiological results and specific clinical features of a particular case. Studies that used this ap- 
proach have demonstrated a decrease in antimicrobial usage and decrease in number of antimicrobials based prescription [22] [23]. The back-end approach provides a benefit in providing capability to emphasis on de-escalation, a factor that is very critical in appropriate antimicrobial usage. De-escalation is amendment of the initial empiric antimicrobial depending upon information from culture test, laboratory results, and patient's clinical status. De-escalation contains altering a broad-spectrum antibiotic to the one with narrower coverage, moving from combined therapy to single therapy, or even stemming antibiotic medication completely as soon as it is evident that these antimicrobial drugs are no more required.

A study was conducted to determine effectiveness of the ASP in significantly improving patient outcomes (e.g. decrease morbidity and mortality from infection). This was undertaken by collecting restrospective data on patient length of hospital stay, bed turnover rate and antibiotic consumption over a period of 2 years since the ASP was initiated. Periodic data was then compared and interpreted based on its clinical, quality and financial implications in order to measure effectiveness of the program implementation.

\section{Materials and Methods}

An ASP was initiated in Ahmadi Hospital to assess antimicrobial usage, suitability of antibiotic selection and appropriateness of utilization. Different clinical departments and multi-disciplinary experts were involved and a complete set-up of ASP was designed.

Hospital-wide ASP educational sessions and campaigns were facilitated. Core contributors in the ASP such as doctors, pharmacists, infection preventionists, medical technologists, nurses and Information Technology (IT) specialists were prioritized in the engagement sessions which focused on the prudent and appropriate use of antibiotics.

Available data on antibiotic prescriptions were analyzed. Following this, a rationalization was applied in the process of ordering each type of antibiotic for all clinical specialties to control prescriptions and prevent inappropriate use. A specialty-specific antibiotic policy was then implemented for each clinical department to comply with. This policy specified antibiotics to be used for each type of infection, clinical condition and pathogen. Antibiotics were selected based on the annual hospital antibiogram report.

An infection control surveillance was also activated to look into the efficacy, side effects, dosage adjustment and alteration of antibiotics. Special focus was given to the development of antibiotic-induced Clostridium difficile infection in inpatients.

Data on average length of stay and bed turnover were collected. These were gathered over a period of 2 years (2018-2019) in two same quarters, April to June. In addition to this, antibiotic consumption data was also obtained from the pharmacy. Data was categorized into 1) no. of antibiotic prescriptions per specialty and 2) No. of antibiotic prescriptions by drug name and type. All data col- 
lected were analyzed using SPSS-23 and correlated to evaluate changes in trends during the ASP implementation.

\section{Results}

\subsection{Hospital-Related Results}

\subsubsection{Average Length of Stay (ALOS)}

The Average Length of Stay (ALOS) was calculated by dividing the sum of lengths of stay for all discharged patients (including deaths) into the total number of discharges (including deaths) and is expressed as hospital or calendar days. Comparing the data from 2018 to 2019, it can be inferred that there is an increase on the ALOS from 4.4 to 5.1 hospital days. Figure 1 shows ALOS in 2019 was higher in all three months compared to the same period in 2018. There is a marginal difference between the ALOS on the month of May of the years in comparison, from 4.3 to 5.4 hospital days making it the month with the most significant change in trend. Though also increased by 0.5 days, April remained with the minimal ALOS in all three months.

\subsubsection{Bed Turnover Rate (BTR)}

Bed Turnover Rate (BTR) was calculated by dividing the number of discharges (including deaths) in a given period and the hospital bed count during the period. The result is then expressed as cycles or discharges per bed per period. Figure 2 shows a change in the trend pattern between 2018 and 2019. Bed turnover in April was nearly twice as the rate in the preceding year. It also illustrates

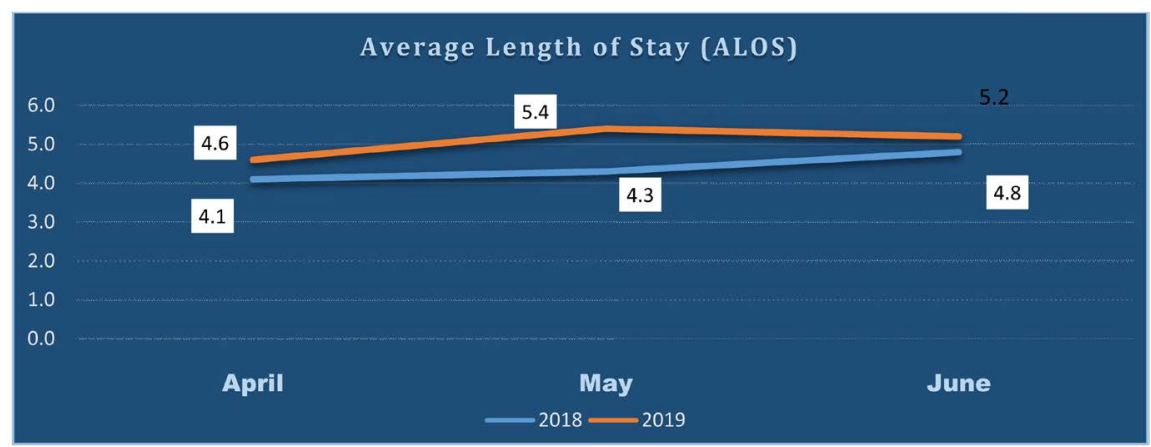

Figure 1. Average length of hospital stay from April to June in 2018 and 2019.

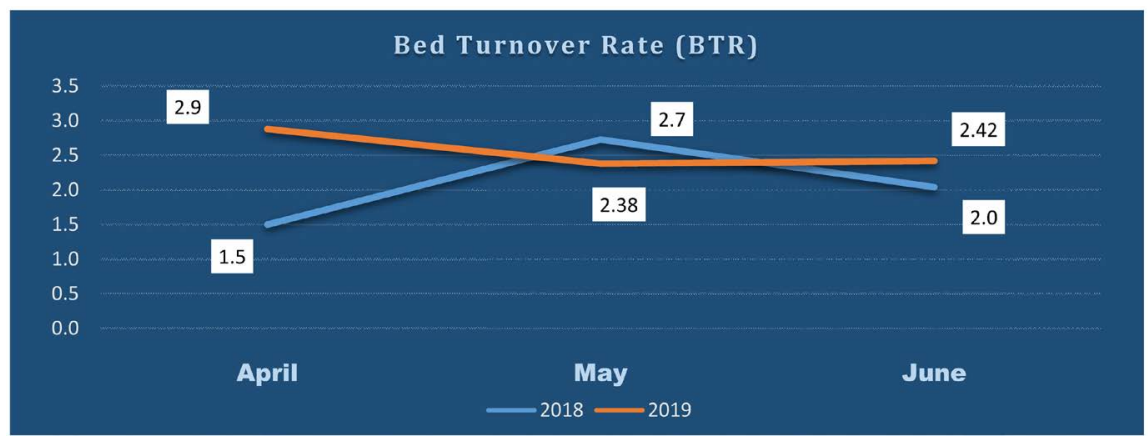

Figure 2. Hospital bed turnover rate from April to June in 2018 and 2019. 
a change in the trend with the month of May decreasing to 2.38 in 2018 from 2.7 in 2019. The turnover rate in June, on the other hand, increased from 2.0 to 2.42 . Overall, the median BTR is at 2.0 in 2018 and 2.5 in 2019.

\subsection{Antibiotic Consumption}

\subsubsection{Antibiotic Prescription per Clinical Specialty}

Data on the total number of antibiotics prescribed by each specialty was gathered and analyzed by bar plots. From August to December of 2018 (5-month period), a total no. of 4837 prescriptions were noted, averaging 32 prescriptions per day, as illustrated in Figure 3. By March to August of 2019 (6-month period): 5324 prescriptions were recorded, averaging 29 prescriptions per day, as shown in Figure 4 . In both time periods, medical physicians prescribed the most antibiotics. Medical doctors averaged at 14.8 in 2018 which lowered to 12.1 prescriptions in 2019. The surgeons, on the other hand, initially averaged at 4.76 in 2018 and improved at 4.05 the succeeding year. The pediatricians, however, remained on an average of 4.21 prescriptions for both observation periods. The specialty with the least number of prescribed antibiotics were the dentists.

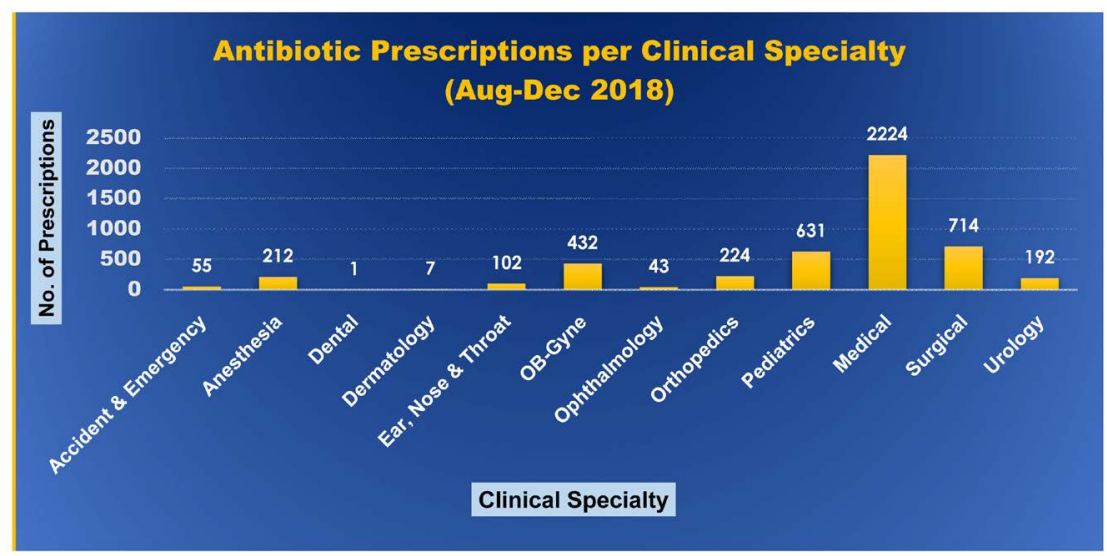

Figure 3. Number of antibiotic prescriptions per clinical specialty from August to December 2018.

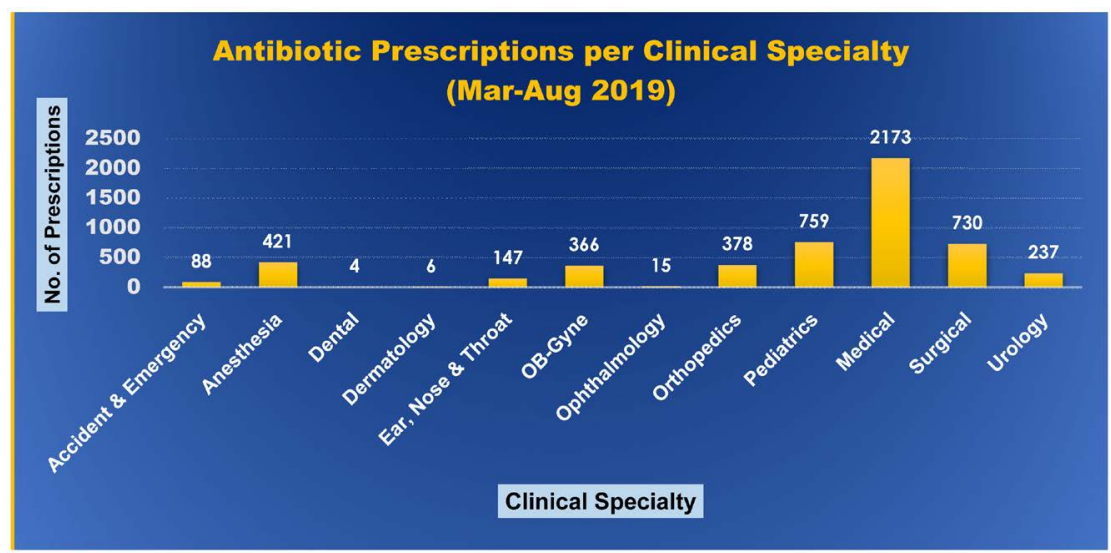

Figure 4. Number of antibiotic prescriptions per clinical specialty from March to August 2019. 


\subsubsection{Antibiotic Consumption per Medication}

Both time periods in Figure 5 and Figure 6 show that Ceftriaxone and Piperacillin/Tazobactam were the most frequently prescribed antibiotics. Ceftriaxone tallied to more than a thousand prescriptions followed by Piperacillin/Tazobactam with about half as many as the latter. Other antibiotics that are mostly ordered for patients were Tavanic (Levofloxacin), Metronidazole, Meronem (Meropenem) and Dalacin-C (Clindamycin).

\section{Discussion}

In this study, ALOS has increased in 2019 as compared to 2018 (Figure 1). This implies that patients are staying in the hospital for a longer period of time as compared to the previous year. Indications of higher ALOS are slow recovery of the patients and slow decimation of the disease-causing organisms. This may be due to microbes developing resistance and requiring longer exposure to the antibiotics for full recovery from the disease. Hence, resulting in longer inpatient admissions, various studies have concluded increase in resistance to several

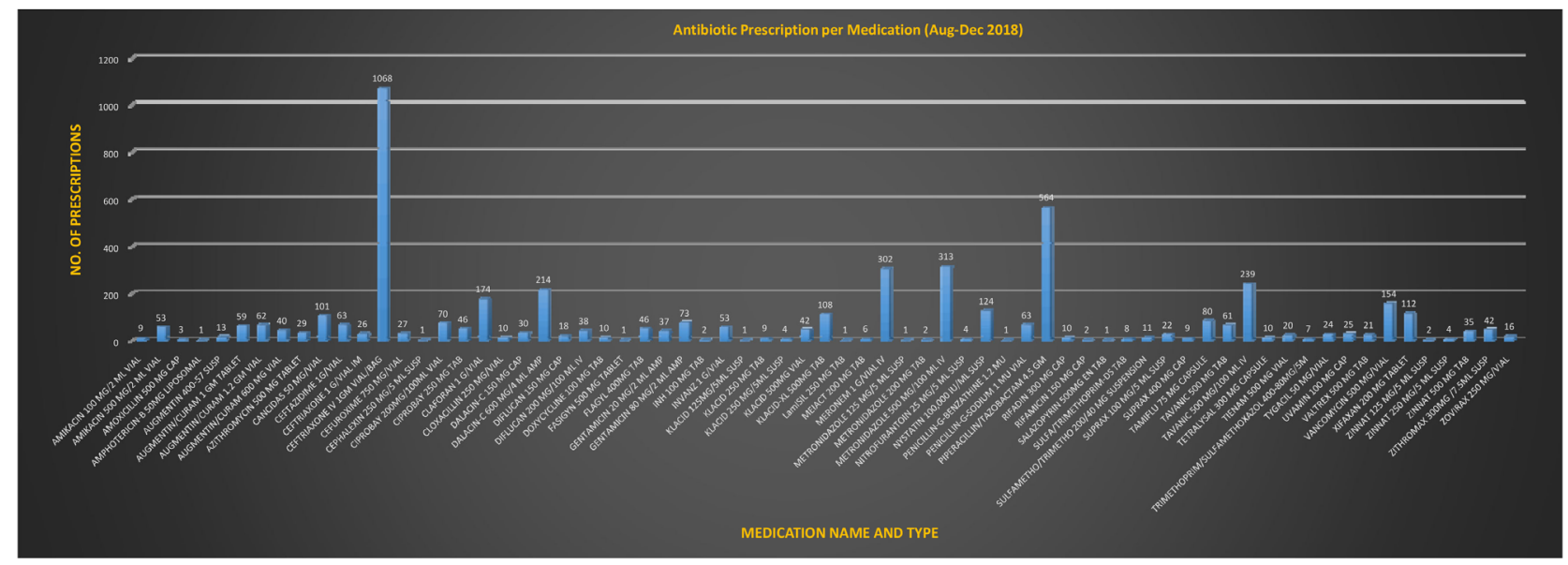

Figure 5. Number of antibiotic prescriptions per medication from August to December 2018.

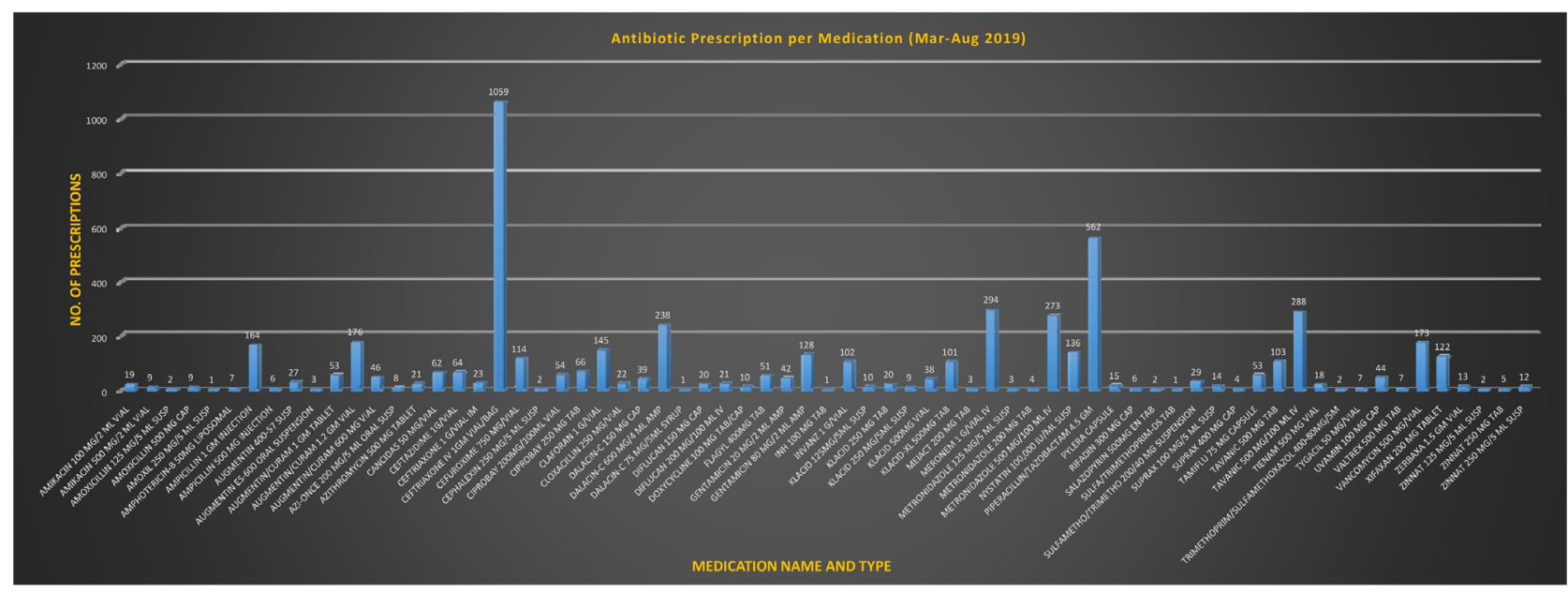

Figure 6. Number of antibiotic prescriptions per medication from March to August 2019. 
antibiotics such as Piperacillin [24]. This antibiotic has been majorly used in our institution (Figure 5 and Figure 6). This implicates avoidance of inappropriate usage of antibiotics in the future to prevent drug resistance.

An increase in the median bed turnover rates (Figure 2) may have been brought about by the facility transition. This occurred in the next two years following mobilization of old patients and admission of new cases due to the increase in bed capacity. Similarity in the patterns of admissions, discharges and BTR validates the concept that as the number of admissions and discharges are increasing, a high turnover rate is expected. Corresponding facilities and services are enhanced to tackle the increasing number of inpatients. This happened as a result of starting of a systematic antibiotic stewardship plan in the hospital [14].

Several studies have suggested decreasing antibiotic usage in the children by parents awareness and interventions in the provision of antibiotics [25]. In our setting, pediatricians are among the top three physician specialties with the most number of prescriptions (Figure 3 and Figure 4). Prudent use implies minimal possible use of antibiotics especially in children [26].

Studies have demonstrated that increased usage of Ceftriaxone has resulted in resistance, giving rise to Penicillin-resistant Streptococcus pneumonia. The increased usage of Ceftriaxone has resulted in several alterations in the penicillin-binding targets in $S$. pneumonia [27]. In our setting, Ceftriaxone has maximum usage among all the antibiotics listed with a big marginal difference (Figure 5 and Figure 6). Several other antibiotics that are oftenly used in our hospital are Tavanic (Levofloxacin), Metronidazole, Meronem (Meropenem) and Dalacin-C (Clindamycin) (Figure 5 and Figure 6). Several studies have suggested resistance against these agents [28] [29]. There is dire need for the appropriate use of these antibiotics to stem the mechanism of antibiotic resistance evolution.

Commitment and cooperation of several hospital departments is paramount to the smooth execution of the ASP. Strategies include proper education, working in collaboration with pharmacists and medicine experts, and optimizing dosage of the antibiotic, which are all interdependently important.

\section{Funding Information}

No funding was received for this study. S. M. A. and W. N. A. are full-time employees of Ahmadi Hospital and preparation of this manuscript was entirely selffunded.

\section{Conflicts of Interest}

All authors declared no competing interests for this work.

\section{References}

[1] Gould, I.M. and Bal, A.M. (2013) New Antibiotic Agents in the Pipeline and How They Can Help Overcome Microbial Resistance. Virulence, 4, 185-191. https://doi.org/10.4161/viru.22507 
[2] Piddock, L.J.V. (2012) The Crisis of No New Antibiotics-What Is the Way Forward? The Lancet Infectious Diseases, 12, 249-253. https://doi.org/10.1016/S1473-3099(11)70316-4

[3] Rossolini, G.M., Arena, F., Pecile, P. and Pollini, S. (2014) Update on the Antibiotic Resistance Crisis. Current Opinion in Pharmacology, 18, 56-60. https://doi.org/10.1016/j.coph.2014.09.006

[4] Spellberg, B. and Gilbert, D.N. (2014) The Future of Antibiotics and Resistance: A Tribute to a Career of Leadership by John Bartlett. Clinical Infectious Diseases, 59, S71-S75. https://doi.org/10.1093/cid/ciu392

[5] Sengupta, S., Chattopadhyay, M.K. and Grossart, H.-P. (2013) The Multifaceted Roles of Antibiotics and Antibiotic Resistance in Nature. Frontiers in Microbiology, 4, 47. https://doi.org/10.3389/fmicb.2013.00047

[6] Kumarasamy, K.K., Toleman, M.A., Walsh, T.R., Bagaria, J., Butt, F., Balakrishnan, R., Chaudhary, U., Doumith, M., Giske, C.G. and Irfan, S. (2010) Emergence of a New Antibiotic Resistance Mechanism in India, Pakistan, and the UK: A Molecular, Biological, and Epidemiological Study. The Lancet Infectious Diseases, 10, 597-602. https://doi.org/10.1016/S1473-3099(10)70143-2

[7] Cosgrove, S.E., Kaye, K.S., Eliopoulous, G.M. and Carmeli, Y. (2002) Health and Economic Outcomes of the Emergence of Third-Generation Cephalosporin Resistance in Enterobacter Species. Archives of Internal Medicine, 162, 185-190. https://doi.org/10.1001/archinte.162.2.185

[8] This Week (2013) The Antibiotic Alarm. Nature, 495, 141. https://doi.org/10.1038/495141a

[9] Gonzales, R., Malone, D.C., Maselli, J.H. and Sande, M.A. (2001) Excessive Antibiotic Use for Acute Respiratory Infections in the United States. Clinical Infectious Diseases, 33, 757-762. https://doi.org/10.1086/322627

[10] Marr, J.J., Moffet, H.L. and Kunin, C.M. (1988) From the Infectious Diseases Society of America Guidelines for Improving the Use of Antimicrobial Agents in Hospitals: A Statement by the Infectious Diseases Society of America. The Journal of Infectious Diseases, 157, 869-876. https://doi.org/10.1093/infdis/157.5.869

[11] Van Boeckel, T.P., Gandra, S., Ashok, A., Caudron, Q., Grenfell, B.T., Levin, S.A. and Laxminarayan, R. (2014) Global Antibiotic Consumption 2000 to 2010: An Analysis of National Pharmaceutical Sales Data. The Lancet Infectious Diseases, 14, 742750. https://doi.org/10.1016/S1473-3099(14)70780-7

[12] Ventola, C.L. (2015) The Antibiotic Resistance Crisis: Part 1: Causes and Threats. P \& T: A Peer-Reviewed. Journal for Formulary Management, 40, 277-283.

[13] Lesprit, P. and Brun-Buisson, C. (2008) Hospital Antibiotic Stewardship. Current Opinion in Infectious Diseases, 21, 344-349. https://doi.org/10.1097/QCO.0b013e3283013959

[14] Fishman, N. (2006) Antimicrobial Stewardship. American Journal of Infection Control, 34, S55-S63. https://doi.org/10.1016/j.ajic.2006.05.237

[15] Dik, J.-W.H., Vemer,P., Friedrich, A.W., Hendrix, R., Lo-Ten-Foe, J.R., Sinha, B. and Postma, M.J. (2015) Financial Evaluations of Antibiotic Stewardship Programs-A Systematic Review. Frontiers in Microbiology, 6, 317. https://doi.org/10.3389/fmicb.2015.00317

[16] Baur, D., Gladstone, B.P., Burkert, F., Carrara, E., Foschi, F., Döbele, S. and Tacconelli, E. (2017) Effect of Antibiotic Stewardship on the Incidence of Infection and Colonisation with Antibiotic-Resistant Bacteria and Clostridium Difficile Infection: 
A Systematic Review and Meta-Analysis. The Lancet Infectious Diseases, 17, 9901001. https://doi.org/10.1016/S1473-3099(17)30325-0

[17] Paskovaty, A., Pflomm, J.M., Myke, N. and Seo, S.K. (2005) A Multidisciplinary Approach to Antimicrobial Stewardship: Evolution into the 21st Century. International Journal of Antimicrobial Agents, 25, 1-10. https://doi.org/10.1016/j.ijantimicag.2004.09.001

[18] Rzewuska, M., Charani, E., Clarkson, J.E., Davey, P.G., Duncan, E.M., Francis, J.J., Gillies, K., Kern, W.V., Lorencatto, F., Marwick, C.A., McEwen, J., Möhler, R., Morris, A.M., Ramsay, C.R., Rogers Van Katwyk, S., Skodvin, B., Smith, I., Suh, K.N. and Grimshaw, J.M. (2019) Prioritizing Research Areas for Antibiotic Stewardship Programmes in Hospitals: A Behavioural Perspective Consensus Paper. Clinical Microbiology and Infection, 25, 163-168. https://doi.org/10.1016/j.cmi.2018.08.020

[19] Doron, S. and Davidson, L.E. (2011) Antimicrobial Stewardship. Mayo Clinic Proceedings, 86, 1113-1123. https://doi.org/10.4065/mcp.2011.0358

[20] Seligman, S.J. (1981) Reduction in Antibiotic Costs by Restricting Use of an Oral Cephalosporin. The American Journal of Medicine, 71, 941-944. https://doi.org/10.1016/0002-9343(81)90304-1

[21] Hayman, J.N. and Sbravati, E.C. (1985) Controlling Cephalosporin and Aminoglycoside Costs through Pharmacy and Therapeutics Committee Restrictions. American Journal of Health-System Pharmacy, 42, 1343-1347. https://doi.org/10.1093/ajhp/42.6.1343

[22] Fraser, G.L., Stogsdill, P., Dickens Jr., J.D., Wennberg, D.E., Smith Jr., R.P. and Prato, B.S. (1997) Antibiotic Optimization: An Evaluation of Patient Safety and Economic Outcomes. JAMA Internal Medicine, 157, 1689-1694. https://doi.org/10.1001/archinte.1997.00440360105012

[23] Solomon, D.H., Van Houten, L., Glynn, R.J., Baden, L., Curtis, K., Schrager, H. and Avorn, J. (2001) Academic Detailing to Improve Use of Broad-Spectrum Antibiotics at an Academic Medical Center. JAMA Internal Medicine, 161, 1897-1902. https://doi.org/10.1001/archinte.161.15.1897

[24] Paramythiotou, E., Lucet, J.-C., Timsit, J.-F., Vanjak, D., Paugam-Burtz, C., Trouillet, J.-L., Belloc, S., Kassis, N., Karabinis, A. and Andremont, A. (2004) Acquisition of Multidrug-Resistant Pseudomonas Aeruginosa in Patients in Intensive Care Units: Role of Antibiotics with Antipseudomonal Activity. Clinical Infectious Diseases, 38, 670-677. https://doi.org/10.1086/381550

[25] Finkelstein, J.A., Davis, R.L., Dowell, S.F., Metlay, J.P., Soumerai, S.B., Rifas-Shiman, S.L., Higham, M., Miller, Z., Miroshnik, I., Pedan, A. and Platt, R. (2001) Reducing Antibiotic Use in Children: A Randomized Trial in 12 Practices. Pediatrics, 108, 1-7. https://doi.org/10.1542/peds.108.1.1

[26] Venekamp, R.P., Sanders, S.L., Glasziou, P.P., Del Mar, C.B. and Rovers, M.M. (2015) Antibiotics for Acute Otitis Media in Children. Cochrane Database of Systematic Reviews, No. 6. https://doi.org/10.1002/14651858.CD000219.pub4

[27] Chiu, C.-H., Su, L.-H., Huang, Y.-C., Lai, J.-C., Chen, H.-L., Wu, T.-L. and Lin, T.-Y. (2007) Increasing Ceftriaxone Resistance and Multiple Alterations of Penicillin-Binding Proteins among Penicillin-Resistant \& Streptococcus Pneumoniae \& Isolates in Taiwan. Antimicrobial Agents and Chemotherapy, 51, 3404.

https://doi.org/10.1128/AAC.01563-06

[28] Jang, W.H., Yoo, D.H. and Park, S.W. (2011) Prevalence of and Risk Factors for Levofloxacin-Resistant E. coli Isolated from Outpatients with Urinary Tract Infec- 
tion. Korean Journal of Urology, 52, 554-559.

https://doi.org/10.4111/kju.2011.52.8.554

[29] Smith, A. (2018) Metronidazole Resistance: A Hidden Epidemic? British Dental Journal, 224, 403-404. https://doi.org/10.1038/sj.bdj.2018.221 